\title{
Observations nouvelles sur les trachelifer, larves lucifériformes de Jaxea nocturna.
}

\author{
Par
}

\author{
M. E.-L. Bouvier,
}

Professeur au Muséum d'Histoire Naturelle, Paris.

Avec 11 Figures dans le texte.

Durant mon premier séjour au Laboratoire de Biologie maritime de Plymouth, en qualité de "first Ray Lankester Investigator," j'ai eu la bonne fortune de trouver un certain nombre d'exemplaires de la très curieuse forme larvaire découverte en Ecosse, près d'Arran, par G. Brook (1889) qui lui donna le nom de trachelifer. Ces exemplaires furent capturés au filet Petersen par l'Oithona, bateau du Laboratoire, entre

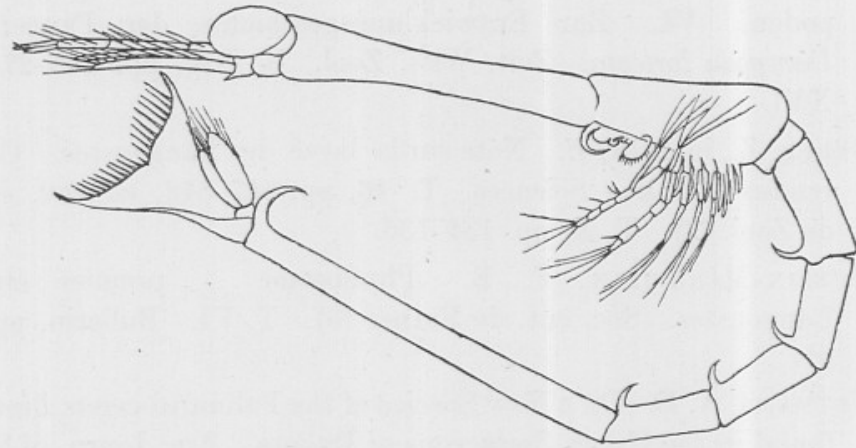

FIG. 1-Trachelifer au stade mysis imparfaite; longueur $9 \mathrm{~mm}$.

Looe et Eddystone, le 13 août 1913. Ils appartiennent sans conteste à la forme même décrite par Brook et, comme eux, présentent les traits suivants qui sont fort caractéristiques (Fig. 1) ; un corps étroit, allongé tout à fait identique à celui des Crustacés décapodes du genre Lucifer; -des yeux volumineux et courts entre lesquels fait saillir une petite pointe rostrale; - des mandibules en forme de long crochet recourbéune paire de crochets épiméraux sur le bord postérieur des segments 2 à 6 de l'abdomen;- - enfin un telson élargi en arrière et muni de soies sur son bord postérieur largement échancré. Brook n'a pas eu de peine à montrer que cette larve ne ressemble aux Lucifer que par sa forme et qu'elle s'en 
distingue par tous les traits essentiels; mais il ne se crut pas en mesure d'émettre une opinion définitive sur ses affinités, d'autant que ses matériaux se limitaient à des individus relativement jeunes, les plus petits mesurant $6 \mathrm{~mm}$. de longeur, les plus grands 9 .

$\mathrm{Au}$ surplus, quelques années avant l'observation de Brook, la même larve avait été découverte dans l'Adriatique par Claus qui, sans lui donner un nom spécial, en fit d'abord $(1884,32)$ connaître deux stades jeunes, l'un de $4 \mathrm{~mm}$. 5, l'autre de $7 \mathrm{~mm} .5$, puis $(1885,63-64)$ un stade beaucoup plus avancé (12 à $15 \mathrm{~mm}$.), ce qui lui permit de regarder cette larve comme celled'un rare Thalassinidé méditerranéen, la Jaxea nocturna, Nardo-Chiereghin (=Calliaxis adriatica Heller).

Depuis, dans un travail fort intéressant au point de vue bibliographique, M. T. Scott $(1898,268-269)$ rapporte qu'il a capturé le trachelifer dans le Golfe de Clyde et a étudié particulièrement un stade avancé dont le longueur atteint $16 \mathrm{~mm}$. 5. La même larve a été retrouvée en abondance par ce zoologiste à Tobermory, détroit de Mull (1901, 481), et par son fils, M. A. Scott (T. Scott, 1901, 481) dans le Barrow Channel, près de Barrowin-Furness, puis plus récemment (1905) dans les mers d'Irlande. Enfin cette larve, au stade le plus jeune, avait été recueillie en Méditerranée et décrite par M. G. Cano, qui en a donné une figuration (1891); d'après A. M. Norman et T. Scott elle aurait également été prise $(1906,13)$ par M. Robert Gurney au large de Salcombe, c'est-à-dire dans les eaux mêmes de la région de Plymouth.

Je dois ajouter, pour être complet, que M. T. Scott $(1900,405)$ a reçu des restes de la forme adulte trouvés dans l'estomac du Rouget ou "gurnard" (Trigla gurnardus L.) et du Pleuronectes cynoglossus, L., dans le Golfe de Clyde ; si bien qu'on doit croire, avec M. Scott, que la forme adulte habite réellement les eaux anglaises.

Là se bornent, à ma connaissance, les observations relatives à la curieuse larve; comme elles sont fragmentaires et éparses, j'ai cru bon de les réunir en les augmentant de celles que j'ai pu faire au laboratoire de Plymouth sur les quelques exemplaires capturés par l'Oithona.

Stades larvaires actuellement connus.-A tous les stades actuellement connus, le trachelifer est franchement lucifériforme, ce qui le distingue nettement des Lucifer qui n'atteignent leur forme spéciale qu'à l'état de jeune immature, ainsi qu'il résulte des belles observations de M. W.-K. Brooks.*

Longueur $4 \mathrm{~mm}$. ( $1^{\mathrm{er}}$ stade larvaire, zoé).-Ce stade a été décrit et par-

* W. K. Brooks. "Lucifer: a Study in Morphology" (Philosoph. Transactions, Vol. 173, p. 57-137, 11 Planches, 1883).

NEW SERIES.-VOL. x. No. 2. JUNE, 1914. 
ticulièrement figuré par Claus (1884, 32, Figs. 48-50). Ses appendices buccaux sont développés, avec la mandibule gauche et le paragnathe en long crochet courbé, caractère tout à fait propre à cette larve et qui persiste à tous les stades; la mandibule droite est normale ; les appendices thoraciques se réduisent aux deux paires de maxillipèdes antérieurs qui sont biramés et fonctionnels, du moins au point de vue de la natation. Le telson est élargi en arrière et très profondément échancré, beaucoup plus que dans les autres stades; il ne porte qu'un petit nombre de soies.

Longueur $6 \mathrm{~mm}$. (zoé).—Stade étudié et figuré par Brook (1889, 420, Fig. 1). Les pédoncules antennulaires paraissent indivises et le fouet qui les termine est remarquablement court; les antennes présentent une épine basilaire et deux branches subégales dont l'externe porte des soies et n'atteint pas l'extrémité distale des pédoncules antennulaires. Les mandibules conservent les traits du stade précédent; les deux paires de mâchoires sont biramées. La troisième paire de maxillipèdes apparaît sous la forme d'un bourgeon simple, comme d'ailleurs les péréiopodes des deux premières paires. Les autres appendices ne sont pas encore développés et aucune ligne articulaire ne sépare le telson du dernier segment abdominal.

Longueur $7 \mathrm{~mm}$. 5 (zoé). - Stade étudié par Claus (1884, 32). Les trois,paires de maxillipèdes sont biramés et fonctionnels ; les péréiopodes sont tous représentés par des bourgeons.

Un stade un peu plus avancé a été décrit par Brook $(1889,420-421)$ : les péréiopodes antérieurs s'allongent en acquérant un exopodite fonctionnel ; les uropodes apparaissent à la base du telson qui est séparé du $6^{\mathrm{e}}$ segment abdominal. Les péréiopodes des deux paires postérieures ne seraient pas encore développés ce qui ne concorde guère avec les observations de Claus et semble pour le moins douteux. Ce stade est intermédiare entre la zoé et la mysis.

Longueur $9 \mathrm{~mm}$. environ-(mysis imparfaite). C'est le dernier stade étudié par Brook et celui auquel appartiennent la plupart des exemplaires de l'Oithona; les caractères schizopodiens sont indiqués par la division en exopodite et endopodite des six paires d'appendices thoraciques antérieurs, mais le stade mysis n'est pas encore complètement réalisé, car les exopodites des cinq paires antérieures fonctionnent seuls comme rames natatoires. Il convient de décrire ce stade dont Brook n'a fait qu'une étude incomplète (1889, 421, Fig. 2).

Les antennules (Figs. 1 et 2) sont longuement pédonculées mais les. trois articles de leur pédoncule semblent peu distincts ; elles se terminent. 
par deux courts fouets simples, l'un externe assez fort, l'autre interne plus long et plus grêle. Il y a de longues soies sur la face dorsale des pédoncules et à l'extrémité distale du fouet externe. Les antennes (Figs. 1 et 2) n'atteignent pas l'extrémité des pédoncules antennulaires ; leur second article présente une pointe antéro-externe et porte à son sommet deux longues branches subégales, simples l'une et l'autre: un exopodite ou écaille, longuement sétifère, un endopodite un peu plus long et dépourvu de soies.

Les appendices buccaux sont, comme dans le genre Lucifer, situés fort loin des yeux, juste derrière l'étranglement qui sépare du thorax la très longue et fort grêle région céphalique (Fig. 1), ils comprennent des

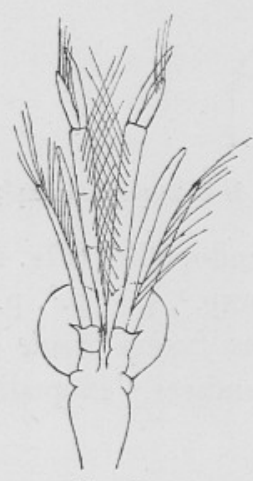

Fig. 2.

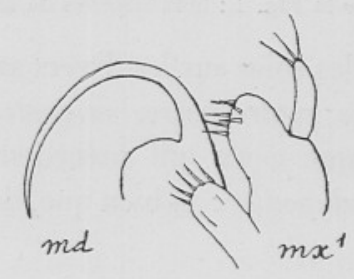

Fig. 3.

Fig. 2,-Trachelifer de la Fig. 1: yeux, antennes et antennules vus du côté dorsal.

Fig. 3.-Trachelifer de la Fig. 1: une mandibule (md), une maxille $\left(\mathrm{mx}^{1}\right)$, et une mâchoire $\left(\mathrm{mx}^{2}\right)$.

mandibules, des maxilles, et des mâchoires (Fig. 3). Les paragnathes et mandibules $(\mathrm{md})$ présentent toujours la forme curieuse signalée par Claus dans les exemplaires du premier stade, à savoir celle d'un crochet longuement recourbé. Je n'y ai pas vu de palpe. L'un des crochets m'a paru simple, l'autre était finement denticulé vers le bout distal. Les maxilles $\left(\mathrm{mx}^{1}\right)$ sont très normales avec un palpe simple muni au sommet de trois soies et une lacinie bien développée mais pauvrement sétifère; je n'y ai pas vu de lame exopodiale. Les mâchoires $\left(\mathrm{mx}^{2}\right)$ ont également une structure normale; leurs lacinies externe et interne sont armées de soies, mais indivises; leur palpe est court, leur exopodite cilié. Je n'ai pu observer la partie postérieure de cette lame exopodiale.

Les appendices thoraciques (Figs. 1, 4, et 5) sont au nombre de huit paires. Les deux paires antérieures (Fig. 4, maxillipèdes 1 et 2 ) se différencient de toutes les autres (Fig. 5) en ce sens que l'article basilaire de leur exopodite est fusionné complètement avec l'article basal de l'endopodite, 
d'où il résulte que la partie libre de l'exopodite se compose seulement de deux articles et la partie libre de l'endopodite de quatre; le dernier article de l'exopodite se termine par six longues soies bipennées; on
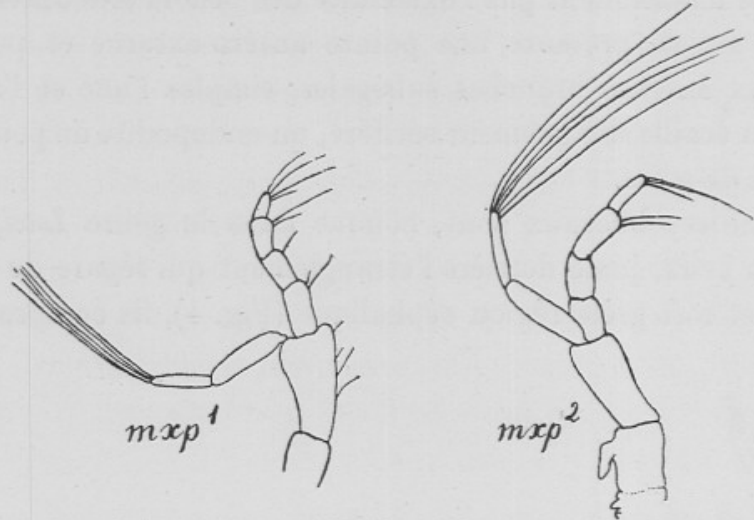

Fig. 4. -Trachelifer de la Fig. 1 : maxillipèdes de la $1^{\text {re }}\left(\mathrm{mxp}^{1}\right)$ et de la $2^{\mathrm{c}}$ paires $\left(\mathrm{mxp}^{2}\right)$

trouve également des soies sur les divers articles de l'endopodite (Fig. 4). Les appendices des quatre paires suivantes (Fig. 5, mxp. ${ }^{3}, \mathrm{p}^{1},{ }^{1}, .^{2},{ }^{2} .{ }^{3}$ ) sont biramés comme ceux qui précèdent, mais sans fusion basale de l'exopodite et de l'endopodite; si bien que, dans ces appendices, l'exopodite

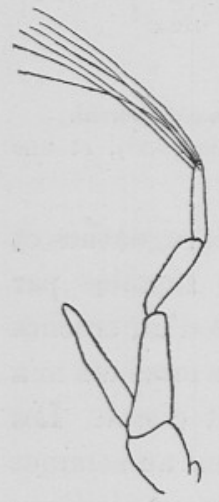

$m x p^{3}$

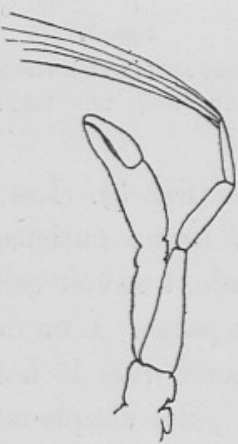

$p^{\prime}$

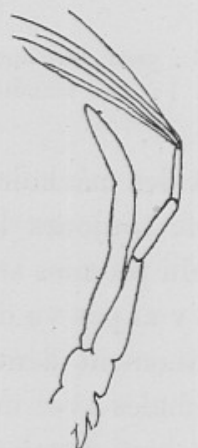

$p^{2}$

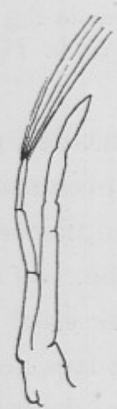

$p^{3}$
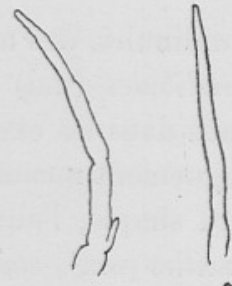

$p^{5}$

FIG. 5. -Trachelifer de la Fig. 1: maxillipède postérieur et les cinq péréiopodes du même côté $\left(\mathrm{p}^{1}\right.$ à $\left.\mathrm{p}^{5}\right)$.

se compose de trois articles libres. Au surplus, l'exopodite n'est sétifère et fonctionnel que dans les trois paires antérieures (maxillipèdes 3, péréiopodes 1 et 2), il se réduit à l'état de bourgeon dans ceux de la dernière paire ( $6 \mathrm{e}$ paire thoracique representant les péréiopodes 3 ) comme d'ailleurs l'endopodite des quatre paires qui nous occupent. J'ajoute 
que ces bourgeons endopodiaux sont simples (Fig. 5), sans articulation apparente, toujours dépourvus de soies, très courts dans les appendices antérieurs, plus allongés en arrière où ils atteignent à peu près les dimensions de l'exopodite. Les appendices des deux paires postérieures (péréiopodes 4 et 5) sont réduits à l'état de bourgeons simples, privés de soies et non fonctionnels, ceux de la dernière paire étant un peu plus courts que les précédents (Fig. 5, p. ${ }^{4}, \mathrm{p} .{ }^{5}$ ).

Ainsi le thorax des larves à ce stade porte six paires d'appendices biramés dont la sixième n'est pas fonctionelle et dont les deux premières se différencient de toutes les autres par la fusion basale de leurs exopodites et endopodites qui sont l'un et l'autre fonctionnels; à partir de la troisième paire, les endopodites se présentent sous la forme de bourgeons non fonctionnels, comme les appendices des deux dernières paires qui, d'ailleurs, ne sont pas biramés.

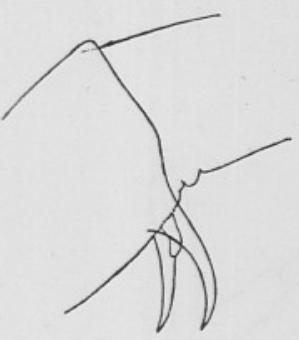

Fig. 6.

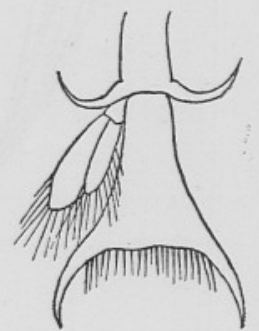

Fig. 7.

FIG. 6. -Trachelifer de la Fig. 1 : le $7^{\mathrm{c}}$ segment abdominal avec ses épines postérieures et ses bourgeons pléopodiaux.

Frg. 7.-Trachelifer de la Fig. 1: dernier segment abdominal, telson et uropode gauche.

Cette description des appendices thoraciques diffère beaucoup de celle qu'a donnée Brook; car mon regretté prédécesseur n'a signalé ni la bifurcation des péréiopodes, ni la curieuse particularité des deux paires de maxillipèdes antérieures. Et ces deux caractères sont, à coup sûr, les plus importants dans la forme que nous étudions. J'ajoute que Brook mentionne simplement, sans les décrire, les maxilles et les mâchoires.

L'abdomen (Figs. 1, 6, 7) présente les caractères signalés par Brook, avec de longues pointes épimérales (nulles sur le premier segment, particulièrement longues sur le sixième) et un telson très élargi en arrière où il présente 11 ou 12 paires de soies spiniformes (Fig. 7). La caractéristique du stade dont nous faisons l'étude, c'est l'apparition des uropodes (Fig. 7) qui se composent de deux lames sétifères subégales beaucoup plus courtes que le telson. Les pléopodes font défaut ou, dans quelques cas très rares, apparaissent sous la forme d'un très court bourgeon (Fig. 6). 
Longueur 10 à $11 \mathrm{~mm}$. (mysis).-Un exemplaire capturé par l'Oithona diffère des précédents en ce sens que les exopodites des appendices thoraciques de la Ce paire (péréiopodes 3 ) sont fonctionnels et que l'endopodite des péréiopodes de la paire antérieure commence à se fendre pour produire la pince (Fig. 8). C'est le stade mysis proprement dit.

Longueur $13 \mathrm{~mm}$. (stade mysis à chélipèdes).-Ce stade n'a pas été signalé jusqu'ici; il est représenté dans les récoltes de l'Oithona par un seul individu. Cet exemplaire ressemble tout à fait à ceux qui précédent par la structure du corps, mais il s'en distingue par les caractères suivants, dont certains ont une grande importance.

Les pédoncules antennulaires sont fort nettement triarticulés; les antennes présentent une épine antéro-inférieure sur chacun de leurs deux

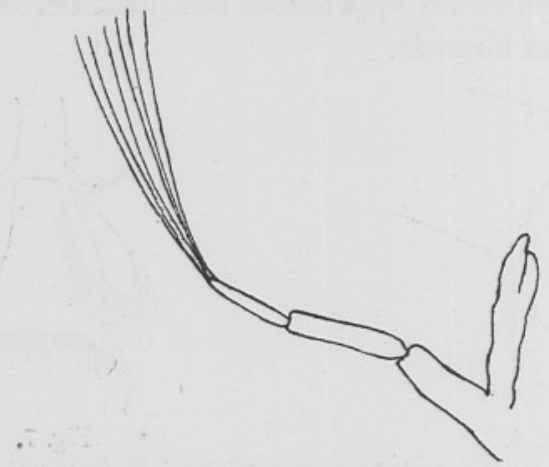

Fig. 8.-Trachelifer au stade mysis, long de $10 \mathrm{~mm}$.: un péréiopode antérieur avec l'endopodite commençant à se transformer en pince.

articles basilaires, leur endopodite se différencie en une hampe basale et un fouet; ce dernier dépasse notablement les antennules mais n'est pas encore brisé en articles.

Les pléopodes abdominaux se développent sur les segments 2 à 5 sous la forme de bourgeons allongés et bifurqués (Fig. 8). Les rames des uropodes restent subégales, et dépassent notablement le milieu du telson. L'échancrure terminale de celui-ci est limitée en avant par un bord presque rectiligne et sur les côtés par les deux pointes normales, qui sont divergentes et presque droites.

Les appendices buccaux et les yeux ne présentent rien de particulier; les appendices thoraciques sont en même nombre qu'au stade précédent, de même type, et doués des mêmes fonctions, les appendices de la $\mathrm{Ce}$ paire (péréiopodes 3) étant semblables aux précédents en ce sens que leur exopodite est sétifère et fonctionnel (Fig. 9 et p. ${ }^{3}$, Fig. 11). Il faut signaler à ce stade le développement d'un très court bourgeon exopodial 
(Fig. 9 et p. ${ }^{4}$, Fig. 11) à la base des appendices de la paire suivante (péré-opodes 4); la présence de cet exopodite rudimentaire montre qu'il faut considérer comme un endopodite les appendices assez réduits et très vaguement articulés qui représentent les deux dernières paires de péréiopodes (Fig. 8 et p. ${ }^{4}$, p..$^{5}$, Fig. 11).

Mais ce qui distingue surtout notre exemplaire et lui donne une signification importante, c'est la structure des.appendices de la $4^{\mathrm{e}}$ paire (Fig. 9 et p. ${ }^{1}$, Fig. 11) qui réprésentent, comme on sait la paire antérieure des péréiopodes des Crustacés décapodes; dans cette paire, l'endopodite se termine par une pince bien formée et présente des lignes articulaires assez nettes; d'ailleurs les deux pinces sont égales et semblables, très

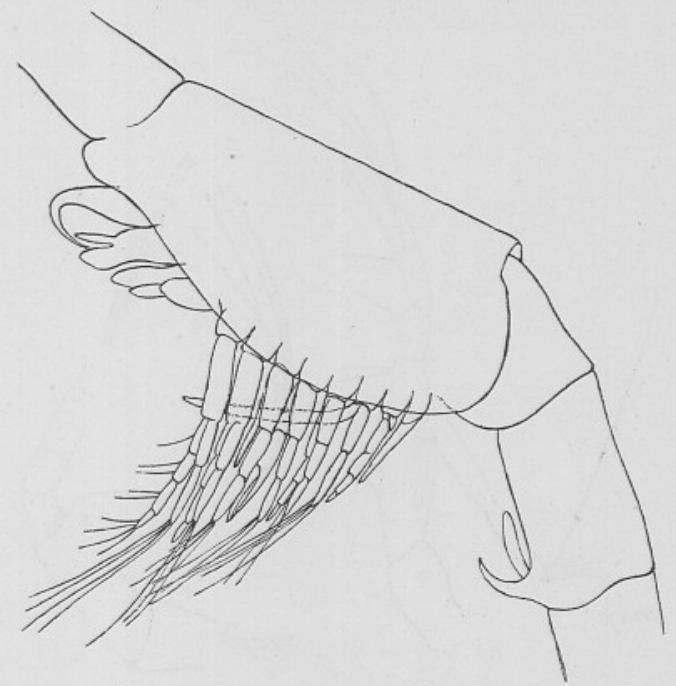

Fig. 9.-Trachelifer au stade mysis à chélipèdes (long. $13 \mathrm{~mm}$.): thorax avec un appendice et partie antérieure de l'abdomen.

développées et à peu près aussi longues que le reste de l'endopodite ; leurs doigts ont à peu près la même longueur que le reste de la portion palmaire et laissent entre eux un léger hiatus.

J'ajoute qu'on observe des bourgeons branchiaux sur tous les appendices thoraciques, depuis la deuxième paire jusqu'à la septième (Figs. 10 et 11).

Longueur 15 à $16 \mathrm{~mm}$. (dernier stade mysis). Je n'ai pas eu d'exemplaires à ce stade qui a été étudié par Claus (1885, 63-64, Taf. V., Fig. 45) avec le plus grand soin, du moins en ce qui regarde la région thoracique. C'est un stade mysis, comme les deux précédents.

Ce stade diffère de celui que je viens de décrire par la segmentation très nette des appendices thoraciques, par la réduction plus grande des 
deux paires postérieures de péréiopodes, et par l'allongement de l'exopodite des péréiopodes 4 qui restent non fonctionnels; enfin et surtout par ses branchies qui sont en même nombre que dans les Jaxea et déjà divisées en lamelles branchiales.

Je crois bien qu'il faut rapporter à ce stade l'exemplaire de $16 \mathrm{~mm}$. brièvement décrit et figuré par M. T. Scott (1898, 269, Pl. 12, Figs. 16-20). A vrai dire, M. Scott ne mentionne pas les branchies mais l'examen qu'il fit de soń exemplaire fut certainement très rapide, si j'en juge d'après les Figures 19 et 16 du travail, qui sont défectueuses.

Conclusions.-M. Scott rappelle, dans son intéressante note, tous les travaux relatifs à la curieuse larve que nous venons d'étudier. Son

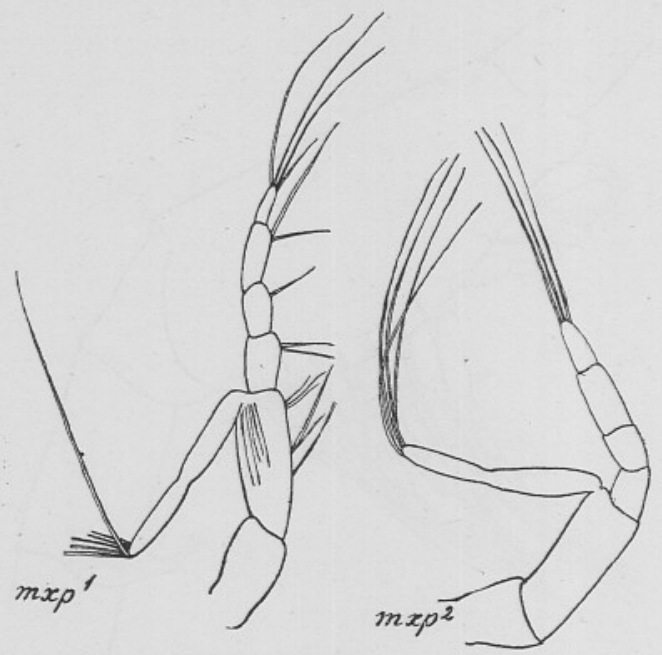

Fig. 10.-Trachelifer de la Fig. 9 : les maxillipèdes des deux premières paires.

travail, à ce point de vue, offre au vif intérêt, et je dois remercier mon excellent collègue M. Calman qui, par l'intermédiaire de M. Allen, a eu l'obligeance de me le faire connaître. Sans cet avis précieux, je me serais borné peut-être à faire une comparaison entre les stades que j'ai décrits et ceux observés par Brook, alors que cet auteur n'a pas reconnu les affinités de sa larve trachelifer et qu'il ignorait, non seulement le travail où Claus a pour la première fois signalé cette larve (1884), mais en outre celui où l'éminent zoologiste de Vienne donna une exacte signification de sa découverte (1885). Or, cette dernière étude est de grande importance: Claus a observé, en effet, que la bizarre larve capturée à Trieste présente, en dépit de sa forme, tous les caractères essentiels des larves des Thalassinidés, qu'elle ressemble tout à fait, par ses caractères, aux 
larves de Gebia littoralis Risso supérieurement étudiées par M. G. O. Sars, * qu'elle possède le même nombre de branchies que le Calliaxis adriatica et qu'il faut la considérer comme la forme larvaire de ce Thalassinien.

Cette identification ne saurait faire le moindre doute; $\uparrow$ elle peut même être poussée jusqu'au détail, car dans les figures données par M. G. O. Sars, on voit que les exopodites des maxillipèdes des deux paires antérieures se distinguent des autres par la fusion de leur article basal avec le deuxième article de l'endopodite. Nous avons montré plus haut qu'il en est de même dans le trachelifer. J'ajoute, comme Claus l'a d'ailleurs observé, que les trachelifer au stade mysis avancé sont dépourvus de pléopodes sur le segment abdominal antérieur et que leurs péréiopodes des deux dernières paires restent à l'état de bourgeons non fonctionnels, deux caractères également propres aux mysis de Gebia.
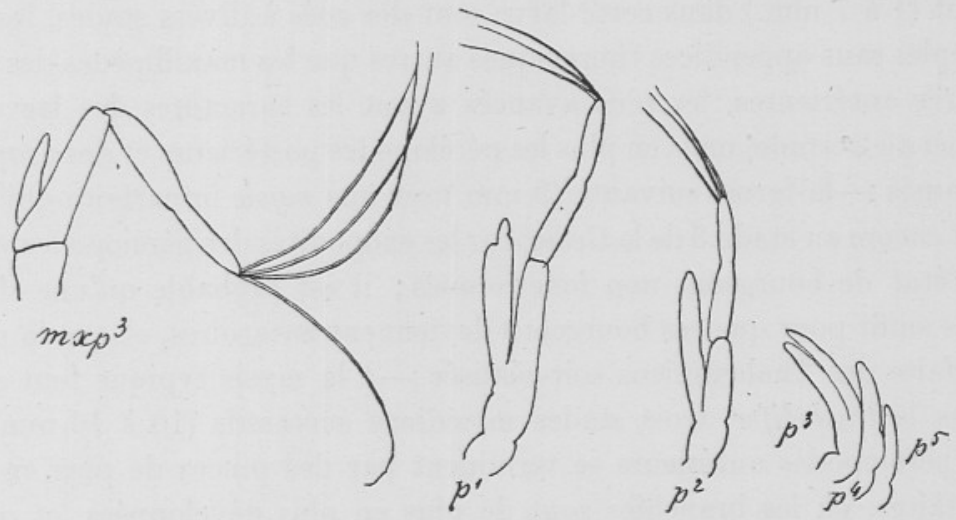

Fig. 11.-Trachelifer de la Fig. 9 : maxillipède postérieur et les cinq péréiopodes du même côté.

M. G. O. Sars signale cinq stades larvaires dans le développement de la Gebia littoralis ; $1^{\circ}$, au premier stade, qui est celui de zoé, les deux paires antérieures de maxillipèdes sont fonctionnelles, et l'on observe les bourgeons, parfois bifurqués, de presque tous les appendices thoraciques suivants, sauf ceux de la dernière paire $; 2^{\circ}$, la deuxième forme larvaire tient le milieu entre la zoé et la mysis; tous les appendices thoraciques sont développés et tous, sauf ceux des deux dernières paires, ont une branche endopodiale et une branche exopodiale, mais cette dernière n'est fonctionnelle que dans les quatre paires antérieures (les

* G. O. Sars. "Bidrag til Kundskab on Decapodernes Forvandlinger. I. Nephrops, Calocaris, Gebia " (Arch. for Math. og Naturvid., B. IX, pp. 155-204, Pls. 1-7, 1884).

+ Elle est d'ailleurs acceptée par M. M. Korschelt et Heider qui ont identifié (1892, 471) le trachelifer avec la larve de Claus, par M. Scott $(1899,69)$ et par M. Calman (1909, 301). 
trois paires de maxillipèdes et les péréiopodes 1$) ; 3^{\circ}$, la troisième forme larvaire correspond au stade mysis, mais c'est une mysis dépourvue d'exopodites sur les péréiopodes des deux dernières paires, les exopodites des autres appendices thoraciques étant d'ailleurs fonctionnels; il y a des bourgeons de pléopodes, le telson est séparé du $6^{\mathrm{e}}$ segment abdominal qui porte en arrière des uropodes biramés; $4^{\circ}$, au dernier stade larvaire, la structure est à peu près identique, mais les pinces commencent à présenter une échancrure digitale et les pléopodes sont allongés avec une seule branche. Au stade suivant, la forme Gebia est réalisée.

Tels sont les quatre stades larvaires indiqués par M. G. O. Sars; on doit les considérer comme des stades essentiels reliés entre eux par des intermédiaires qui se manifestent à la suite de mues. Du moins en est il ainsi dans notre trachelifer:-les trois premières formes décrites plus haut (4 à $7 \mathrm{~mm}$.) dans cette larve sont des zoés à divers stades, les plus simples sans appendices thoraciques autres que les maxillipèdes des deux paires antérieures, les plus avancés ayant les caractères des larves de Gebia au ¿e stade, mais en plus les péréiopodes postérieurs et des uropodes biramés :- la forme suivante $(9 \mathrm{~mm}$.) est une mysis imparfaite qui n'est pas encore au stade 3 de la Gebia, car les exopodites des péréiopodes y sont à l'état de bourgeons non fonctionnels; il est probable qu'une simple mue suffit pour que ces bourgeons deviennent natatoires, et que la mysis parfaite des Thalassiniens soit réalisée;-à la mysis typique font suite, dans le trachelifer, trois stades mysidiens successifs (10 à $16 \mathrm{~mm}$.) cù les péréiopodes antérieurs se terminent par des pinces de plus en plus parfaites, où les branchies sont de plus en plus développées, et où les pléopodes acquièrent progressivement leurs deux rames, le premier de ces stades mysidiens à pinces correspond à très peu près au $4^{\mathrm{e}}$ stade larvaire décrit par Sars dans la Gebia littoralis.

Ainsi, Claus avait amplement raison de regarder la forme larvaire qui nous occupe comme une larve de Thalassinidé, et sans doute aussi avait-il raison de voir dans cette forme curieuse la larve de la Jaxea nocturna; elle ne saurait être rapportée, en effet, aux autres Thalassinidés méditerranéens qui tous ont une forme larvaire plus différente de celle des Gebia; et d'autre part, Claus observe qu'à un stade avancé elle présente les mêmes branchies que la Jaxea nocturna.

Faut-il croire, avec Claus, qu'une mue devra suffire pour conduire de cette larve lucifériforme avancée à l'individu présentant les caractères de l'adulte? J'ai sous les yeux deux exemplaires de Calliaxis adriatica envoyés jadis au Muséum par Heller; ils sont tout à fait d'un type astacien et si différents du trachelifer qu'on pourrait mettre en doute la 
possibilité d'un passage direct de l'un à l'autre. Dans les Thalassinidés comme chez les Paguriens et les Macroures marcheurs cuirassés (Palinuridés, Scyllaridés), un stade intermédiaire doit rattacher la forme adulte à la forme mysidienne, un stade natant (pour me servir d'une terme fort juste emprunté à M. Boas) où la forme se rapproche déjà beaucoup de celle de l'adulte, et où la natation s'effectue au moyen des pléopodes dont l'appendice interne présente des rétinacles. Le jeune de Gebia littoralis figuré par M. G. O. Sars (1884, Taf. V, Fig. 1) est certainement à ce stade intermédiaire, il mesure environ $6 \mathrm{~mm}$.

Le stade natant de la Jaxea nocturna peut-être imaginé un peu plus long et plus grêle, parce que l'adulte diffère de la Gebia littoralis au point de vue de la gracilité du corps; par une contraction et une condensation analogues à celles qui se produisent dans le phyllosome passant au puerulus, le trachelifer lucifériforme long de 15 à $16 \mathrm{~mm}$. donnera un natant plus court de moitié et présentant déjà la forme des Jaxées adultes. Cette forme, d'ailleurs, sera aisément reconnaissable à ses pinces astaciennes, qui sont déjà fort longues dans les mysis et qui le sont plus encore chez l'adulte. Ainsi le petit organisme sera aisé à reconnaître et on le trouvera sans doute quelque jour dans les pêches pélagiques au filet fin.

C'est une capture que l'on peut faire à Plymouth, dans les parages de Looe-Eddystone cù l'Oithona fit l'heureuse trouvaille des exemplaires étudiés dans le présent opuscule. Là également devra-t-on rechercher la forme adulte, Jaxea nocturna, mais alors au moyen de pêches effectués sur le fond, au chalut plutôt qu'à la drague. Cette dernière recherche sera certainement bien plus difficile que la première, car la Jaxée adulte est un animal rarissime, trouvé seulement à Trieste, puis à Naples où d'après S. Lo Bianco $(1898-99,503)$ on n'en put prendre qu'un exemplaire au cours de 25 ans.*

Voilà pour les travailleurs du Laboratoire de Plymouth, un sujet de recherches tout indiqué. Grâce au concours de tout le personnel du Laboratoire, surtout au zèle obligeant de M. le Directeur Allen et de M. Clark, on sait aujourd'hui que la Jaxea nocturna, déjà connue à Salcombe sous la forme de larve, habite aussi le voisinage immédiat de Plymouth. Il n'y a pas lieu de douter qu'on trouvera quelque jour, dans les mêmes eaux, le stade natant inconnu et l'adulte de cette espèce.

* Cet exemplaire fut capturé non loin de la station zoologique, par 15 mètres de profondeur, sur fond de vase et sable fin. 


\section{INDEX BIBLIOGRAPHIQUE.}

1889. G. BRоoк. Notes on a Lucifer-like Decapod Larva from the West Coast of Scotland. (Proc. Royal Soc. Edinburgh, Vol. XV, pp. 420-423, Figs. 1 and 2, 1889.)

1909. W. T. Calman. Appendiculata. Third fascicule. Crustacea, p. 301. (Treatise on Zoology, publié sous la direction de Ray Lankester.)

1891. G. Cano. Sviluppo postembryonale della Gebia, Axius, Callianassa, e Calliaxia. (Boll. Soc. Natur. Napoli, Vol. V, p. 12, Pl. 4, Figs. 1-13, 1891.)

1884. C. Claus. Zur Kentniss der Kreislaufsorgane der Schizopoden und Decapoden. (Arb. Zool. Inst. Wien, B. V, p. 32, Taf. VIII, Figs. 48-50, 1884.)

1885. ID. Neue Beiträge zur Morphologie der Crustaceen (Id., B. VI, pp. 63-64, Taf. V, Fig. 45, 1885.)

1892. E. Korschelt und K. Heider. Lehrbuch der vergleichenden Entwicklungsgeschichte der wirbelloser Thiere. Specieller Theil, p. 471.

1899. S. Lo Bianco. Notizie biologiche riguardente specialemente il periodo di maturata sessuale degli animali del Golfo di Napoli. (Mitth. Zool. Station Neapel, B. XIII. p. 503, 1898-99.)

1906. A. M. Norman and T. Sсотт. The Crustacea of Devon and Cornwall, pp. 12-13, 1906. (Avec toute la bibliographie de l'espèce.)

1905. A. Sсотт. On the Tow-nettings collected in the Irish Sea. (Proc. Liverpool Biol. Soc., T. XIX, pp. 196-205 ).

1899. T. Sсотт. Notes on the Recent Gatherings of Microcrustacea from the Clyde and the Moray Firth. (Annual Report Fishery Board of Scotland, 1898, Part III, pp. 268-269, Pl. 12, Figs. 16-20, 1899.)

1900. In. Notes on some Gatherings of Crustacea collected for the most part on board the Fishery steamer Garland, and examined during the past year (1899). (Eighteenth Annual Report Fishery Board of Scotland, for the year 1899, pp. 405-406, 1900.)

1902. ID. Notes on Gatherings of Crustacea collected by the Fishery steamer Garland and the Steam trawlers Star of Peace and Star of Hope, of Aberdeen, during the year 1901. (Twentieth Annual Report Fishery Board of Scotland, for the year 1901, p. $481,1902$. 